\title{
Function approximation on triangular grids: some numerical results using adaptive techniques
}

\author{
Cristina Manzi, Francesca Rapetti, Luca Formaggia \\ Center of Advanced Studies, Research and Development in Sardinia (CRS4) \\ Via N.Sauro 10, 09123 Cagliari, Italy \\ e-mails: cristina@crs4.it,rapetti@crs4.it,forma@crs4.it \\ 12th February 1998
}

\begin{abstract}
Applications of mesh adaption techniques could be found in the numerical solution of PDE's or in the optimal triangulation of surfaces for shape representation or graphic display. The scope of this work is to verify through numerical experiments the effectiveness of some algorithms for the control of the $L_{\infty}$ error norm for piece-wise linear approximation on 2D unstructured triangular meshes. The analysis could be extended to parametric surfaces and to the 3D case.
\end{abstract}

Keywords: mesh adaption, approximation theory.

\section{Introduction}

Results of interpolation error theory show that, for appropriately smooth functions asymptotically optimal linear triangular elements meshes are controlled by the value of the function Hessian $\mathbf{H}$. Standard results make use of norms of $\mathbf{H}$, reducing all the information to a single scalar value.

However, the full information contained in the Hessian should be employed if one wishes to control not only the size of the elements but also their aspect ratio, with the objective of performig what we will call anisotropic mesh adaption. In the first part of this work we will detail and explain in a different light some results available in the literature for $L_{\infty}$ interpolation error control for piecewise linear interpolation on triangles. We will then illustrate some numerical results and present possible estension. 
First of all, we will give some definitions. Given a function $f(x, y): \mathbf{R}^{2} \rightarrow \mathbf{R}$, its Hessian at point $(x, y)$ is defined as

$$
\mathbf{H}(x, y)=\left[\begin{array}{ll}
\frac{\partial^{2} f(x, y)}{\partial x \partial x} & \frac{\partial^{2} f(x, y)}{\partial x \partial y} \\
\frac{\partial^{2} f(x, y)}{\partial y \partial x} & \frac{\partial^{2} f(x, y)}{\partial y \partial y}
\end{array}\right] .
$$

We will indicate with $\lambda_{1}(x, y)$ and $\lambda_{2}(x, y)$ the maximum and minimum eigenvalues of the absolute value of the Hessian $|\mathbf{H}(x, y)|$, respectively. The ratio $a_{\mathbf{H}}=\lambda_{1} / \lambda_{2}$ will be called the Hessian anisotropy factor. We will say that the Hessian is strongly anisotropic at a point $(x, y)$, if we have $a_{\mathbf{H}}(x, y)>>1$.

We will show that when a function has an high anisotropic Hessian matrix, an $L_{\infty^{-}}$ optimal mesh is obtaind with 'stretched' triangles, recovering some results already found by Rippa[18], and by D' Azevedo and Simpson[4, 5, 19]. Given a computational domain $\Omega$ and a fixed number of elements, we will call the $L_{\infty}$-optimal mesh the one which equidistributes the $L_{\infty}$ norm of the error on the mesh elements. This definition differs from the one which defines the optimal mesh in terms of the maximum error allowed on each element; we will see, however, that for practical purposes, the two definitions are equivalent.

Clearly, the optimality of the mesh depends on the chosen norm. Here we will present results only for the $L_{\infty}$ norm, but results for other norms may be derived following similar arguments. Before proceding further, we want to give a more precise quantification of triangle size and orientation.

Let us consider a triangle $K$, with sides $\mathbf{v}_{K, i}, i=1, \cdots 3$. There is a unique symmetric positive definite matrix $\mathbf{M}_{K}$ so that

$$
\mathbf{v}_{K, i}^{T} \mathbf{M}_{K} \mathbf{v}_{K, i}=1 \quad i=1, \cdots, 3 .
$$

We will call $h_{K, \min }$ and $h_{K, \max }$ the quantities

$$
\left(h_{K, \min }, h_{K, \max }\right)=\left(\mu_{1}^{-\frac{1}{2}}, \mu_{2}^{-\frac{1}{2}}\right),
$$

where $\mu_{1}$ and $\mu_{2}$ are the eigenvalues of $\mathbf{M}_{K}$, with $\mu_{1} \geq \mu_{2}$. Those quantities are measures of the element size. If we indicate with $h_{K}$ the diameter of $K$, then we have that

$$
h_{K, \min } \leq h_{K} \leq h_{K, \max }
$$

We call element aspect ratio the quantity $h_{K, \max } / h_{K, \min }$, which is an indicator of the element anisotropy. The quantity $\mathbf{M}$ may be used to specify the mesh size distribution in mesh generation algorithms[20]. In that case, a continous $\mathbf{M}(\Omega)$ over the domain $\Omega$ is defined, and it induces a Riemannian metric on $\Omega$ where the lenght of $d \mathbf{x}$ is given by

$$
d s^{2}=\left\|d \mathbf{x}^{T} \mathbf{M} d \mathbf{x}\right\|
$$


Then, relation (1) may be used to implicitely define the dimensions of the triangle to be generated, by taking a suitable constant value of $\mathbf{M}$ on each element. Details may be found in $[20,2,3,13]$.

Mesh adaption procedure for anisotropic unstructured meshes could be devised by correlating the error to the metric induced by $\mathbf{M}$. Adaptive mesh strategies which follow this approach for fluid flow computations have been implemented [12, 10, 8, 9] with some success, particularly for compressible flows.

In this work we investigate this type of procedures in a simplified setting. We analyze how an iterative adaption procedure is able to minimize the approximation error for a given function, when the Hessian function is not computed analytically, yet estimated on the last computed triangulation. Numerically, experiments show that, even if no formal proof can be given, the procedure converges to a nearly optimal mesh.

\section{Some interpolation error estimates}

Let consider a finite decomposition of the domain $\Omega \in \mathbf{R}^{2}$ into a conforming mesh of triangles $T_{h}$. It means that the following properties hold:

$$
\begin{gathered}
\bigcup_{K \in T_{h}} K=\bar{\Omega}, \\
K_{i} \cap K_{j}=\emptyset \quad \forall K_{i}, K_{j} \in T_{h}, \quad i \neq j,
\end{gathered}
$$

and every edge of a triangle is either the edge of another triangle of $T_{h}$, or a boundary edge of $\Omega$.

Let $V=\left\{\left(x_{i}, y_{i}\right) \in \mathbf{R}^{2}, i=1, \ldots, N\right\}$ be a set of distinct non collinear data points from a domain $\Omega$. Data interpolation in $\mathbf{R}^{2}$ consists in constructing a function $F_{A}=F_{A}(x, y)$ such that

$$
F_{A}\left(x_{i}, y_{i}\right)=f_{i}, i=1, \ldots, N
$$

where $\left(f_{1}, \ldots, f_{N}\right)$ is a (real) data vector.

We assume that the data vector $\left(f_{1}, \ldots, f_{N}\right)$ is sampled from some underlying smooth function $f(x, y)$ defined on $\Omega$ and we wish to get a good approximation of $f$ by constructing a triangulation $T_{h}$ of $\bar{\Omega}$ and defining the interpolating surface as a linear function $F_{T_{h}, f}$ that takes the same values of $f$ at the vertices of the triangles of $T_{h}$.

The quality of the approximation of $f$ by $F_{T_{h}, f}$ depends on the specific triangulation $T_{h}$, and we would like to choose $T_{h}$ in an "optimal" way using a data-dependent criterion measuring the quality of a triangulation with respect to the $L_{\infty}$ norm of the interpolation error. The construction of optimal triangular meshes to control the quality of the approximation needs two ingredients: an error estimate and a suitable mechanism to recast the mesh according to the estimate. 
Given a triangulation $T_{h}$ of $\bar{\Omega}$, let us consider the interpolation error $\left(f-F_{T_{h}, f}\right)$, defined as the error between the function $f$ and the piecewise linear interpolated function $F_{T_{h}, f}$ of $f$. Classical interpolation error analysis would lead to error estimates on each triangle $K \in T_{h}$ of the kind

$$
\max _{|\alpha|=s}|| D^{\alpha}\left(f-F_{T_{h}, f}\right)\left\|_{L_{\infty}(K)} \leq C h_{K}^{(2-s)} \max _{|\alpha|=2}\right\| D^{\alpha} f \|_{L_{\infty}(K)}, s=0,1
$$

providing that $f \in C^{2}(\bar{\Omega})$ and $T_{h}$ is a regular triangulation, i.e. the angles of the triangles $K$ are not allowed to be arbitrarily small, or, equivalently, their aspect ratio is bounded from above. We note that the size of the interpolation errors in (2) depends on the norm of the Hessian of $f$. An equidistribution of the interpolation error according to the previous formula, that is the imposition of

$$
\left\|f-F_{T_{h}, f}\right\|_{L_{\infty}(K)} \leq c \quad \forall K \in T_{h},
$$

where $c$ is a determined positive constant, would give a condition on the diameter of each triangle $K \in T_{h}$ of the type

$$
h_{K} \leq \sqrt{\frac{c}{2}}\left(\left\|D^{2} f\right\|_{L_{\infty}(K)}\right)^{-\frac{1}{2}}
$$

That estimate does not provide any indication on how the element aspect ratio and orientation may change the error distribution and is suitable for isotropic adaption only. In particular, the bound (2) on the $L_{\infty}$ approximation error (with $s=0$ ) over a triangle $K$ depends only on one length scale.

On the other hand, numerical examples $[4,7]$ have shown that anisotropic meshes may be optimal. In those works, they show cases where a small interpolation error by piecewise linear polynomials is obtained with long and thin triangles. The apparent difference between the expected error and the numerical results can be explained considering that the error bounds (2) contain the implicit assumption that the second derivatives of $f$ are of the same order of magnitude. This assumption may lead to wrong conclusions about the desired shape of a triangle when the function $f$ has an Hessian with a large anisotropy factor.

\section{Anisotropic interpolation error analysis}

Let us consider a triangle $K \in T_{h}$, with centroid $\mathbf{g}_{K}$ and a smooth function $f$ with nondegenerate Hessian in $\mathbf{g}_{K}$. For sake of simplicity and without loss of generality we consider a translation of the origin of the coordinate system on $\mathbf{g}_{K}$. A Taylor expansion around the zero of this new coordinate system will give

$$
f(\mathbf{x})=f_{0}+(\nabla f) \cdot \mathbf{x}+\frac{1}{2} \mathbf{x}^{T}|H| \mathbf{x}+O\left(h_{K}^{2}\right),
$$


where all the derivatives are computed at the origin. The truncation error becomes $O\left(h_{K}^{3}\right)$ if $\mathbf{H}$ is positive definite, in that case, moreover, $|\mathbf{H}|=\mathbf{H}$. Following the developements of D'Azevedo[4], we may write

$$
e_{K}=\left.\left(f-F_{T_{h}, f}\right)\right|_{K}=\left(\mathcal{E}_{K}-\frac{1}{2}\left(\mathbf{x}-\mathbf{x}_{c}\right)^{T}|\mathbf{H}|\left(\mathbf{x}-\mathbf{x}_{c}\right)\right)_{K}+O\left(h_{K}^{2}\right),
$$

where $\mathbf{x}_{c}$ is the center of the zero interpolation error conic whose expression may be found in [5] and

$$
\mathcal{E}_{K}=\frac{\prod_{i=1}^{3} \tilde{\mathbf{v}}_{K, i}}{16 \lambda_{1} \lambda_{2} A_{K}^{2}}
$$

Here,

$$
\tilde{\mathbf{v}}_{K, i}=\mathbf{v}_{K, i}^{T}|\mathbf{H}| \mathbf{v}_{K, i}
$$

while $\lambda_{1}$ and $\lambda_{2}$ are the eigenvalues of $|\mathbf{H}|$ and $A_{K}$ the triangle area. We may then write

$$
\|e\|_{\infty(K)}=\left\|\mathcal{E}_{K}-\frac{1}{2}\left(\mathbf{x}-\mathbf{x}_{c}\right)^{T}|\mathbf{H}|\left(\mathbf{x}-\mathbf{x}_{c}\right)\right\|_{\infty(K)}+O\left(h_{K}^{2}\right) .
$$

We now assume that the centroid $\mathbf{x}_{c}$ falls inside the triangle. In that case the maximum of the quadratic form contained in the error espression is $\mathcal{E}_{K}[5,19]$. Therefore, under those assumptions, the maximum error behaves asimptotically as

$$
\|e\|_{\infty(K)} \approx \frac{\prod_{i=1}^{3} \tilde{\mathbf{v}}_{K, i}}{16 \lambda_{1} \lambda_{2} A_{K}^{2}} .
$$

If we consider the linear trasformation $\mathbf{T}: \mathbf{R}^{2} \rightarrow \mathbf{R}^{2}$, defined by $\mathbf{T}=\sqrt{|\mathbf{H}|}$, it may be immediately recognised that

$$
\lambda_{1} \lambda_{2} A^{2}=A_{\tilde{K}}^{2},
$$

where $A_{\tilde{K}}$ is the area of the trasformed triangle $\tilde{K}=\mathbf{T}(K)$. It is clear that, given a target error $\gamma$, the triangle with the maximum area which satisfies (asimptotically)

$$
\|e\|_{\infty(K)}=\mathcal{E}_{K}=\gamma,
$$

is trasformed by $\mathbf{T}(K)$ into an equilateral triangle, with sides of lenght

$$
\left\|\tilde{\mathbf{v}}_{K, i}\right\|=\sqrt{3 \gamma} .
$$

Consequently, the metric $\mathbf{M}_{K}$ defined in (1), for that triangle would be

$$
\mathbf{M}_{K}=\frac{1}{3 \gamma}|\mathbf{H}| .
$$


An heuristic approach to build an optimal mesh may be devised by exploiting the fact that the area of any transformed optimal triangle is fixed and equal to $\sqrt{3} / 4$. We assume that we use a metric distribution on the domain $\Omega$, given by

$$
\mathbf{M}(x, y)=\frac{1}{3 \gamma}|\mathbf{H}(x, y)| \text {. }
$$

The algorithm would then read as follows:

- given the desired numer of elements on the grid $N_{\Omega}$;

- compute the quantity

$$
I_{\Omega}=\int_{\Omega} \frac{1}{\sqrt{\lambda_{1} \lambda_{2}}} d \Omega
$$

by employing a numerical quadrature, with the help, for example, of a background grid;

- compute the corresponding error by equidistribution as

$$
\gamma=\frac{\sqrt{3}}{12} \frac{N_{\Omega}}{I_{\Omega}} ;
$$

- construct a grid which is optimal with respect to the metric $\mathbf{M}$ given by relation (3).

\section{Mesh adaption}

\begin{tabular}{|r|c|c|}
\hline \hline \multicolumn{3}{|c|}{ L $_{\infty}$ error norm } \\
$f(x, y)=y \cos (x)+\sin (y)+1$ \\
\hline \hline Elements & Uniform & Optimal \\
\hline 500 & $4.6310^{-2}$ & $3.0510^{-2}$ \\
1000 & $2.1510^{-2}$ & $9.1310^{-3}$ \\
2000 & $9.7710^{-3}$ & $9.6010^{-3}$ \\
\hline \hline
\end{tabular}

Figure 1: Approximation error for uniform and optimal triangulations using exact second derivatives.
We have shown that, in accordance with the works of D'Azevedo, Simpson [5] and Vallet [20], an error equidistribution strategy based on approximation error theory for linear elements can be based on a metric which is proportional to the absolute value of the Hessian $\mathbf{H}$, i.e., $\mathbf{M}=k|\mathbf{H}|$, when

$$
k=\frac{1}{3 \gamma}=\frac{4 I_{\Omega}}{\sqrt{3} N_{\Omega}} .
$$

When the analytical function is unknown, there is the additional problem of evaluating the Hessian. To this purpose, we have estimated the second derivatives using the following least-square procedure. 
Let consider the Taylor formula truncated at the first order term

$$
f_{k} \approx f_{i}+(\nabla f)_{i} \cdot\left(\mathbf{x}_{k}-\mathbf{x}_{i}\right),
$$

where $\mathbf{x}_{i} \equiv\left(x_{i}, y_{i}\right)^{T}$ is the vector containing the coordinates of mesh node $i$. A weighted least squares approach allows to compute an approximation to the first derivatives $\nabla \tilde{f} \equiv\left(\tilde{f}_{, x}, \tilde{f}_{, y}\right)$ by solving at each node $i$ a system of the type

$$
\left(A_{i}^{T} W_{i}^{T} W_{i} A_{i}\right)\left[\begin{array}{l}
\tilde{f}_{\tilde{f}_{x}} \\
\tilde{f}_{, y}
\end{array}\right]=A_{i}^{T} W_{i}^{T} W b_{i}
$$

where

$A_{i}=\left[\begin{array}{cc}\Delta_{1} x_{i} & \Delta_{1} y_{i} \\ \vdots & \\ \Delta_{n} x_{i} & \Delta_{n} y_{i}\end{array}\right], \quad b_{i}=\left[\begin{array}{c}\Delta_{1} f_{i} \\ \vdots \\ \Delta_{n} f_{i}\end{array}\right]$

We have indicated with $\Delta_{k} f$ the difference $\left(f_{k}-f_{i}\right)$ of the function at the $k$-th of the $n$ nodes adjacent to $i$ and the function at $i$. The weights can be chosen so that to increase the accuracy of scheme. A second step allows to estimate the second derivatives as follows.

$$
\left(\hat{A}_{i}^{T} \hat{W}_{i}^{T} \hat{W}_{i} \hat{A}_{i}\right)\left[\begin{array}{c}
\tilde{f}_{\tilde{f}} x x \\
\tilde{f}_{\tilde{f}} x y \\
\tilde{f}_{, y y}
\end{array}\right]=\hat{A}_{i}^{T} \hat{W}_{i}^{T} \hat{W}_{i} \hat{b}_{i}
$$
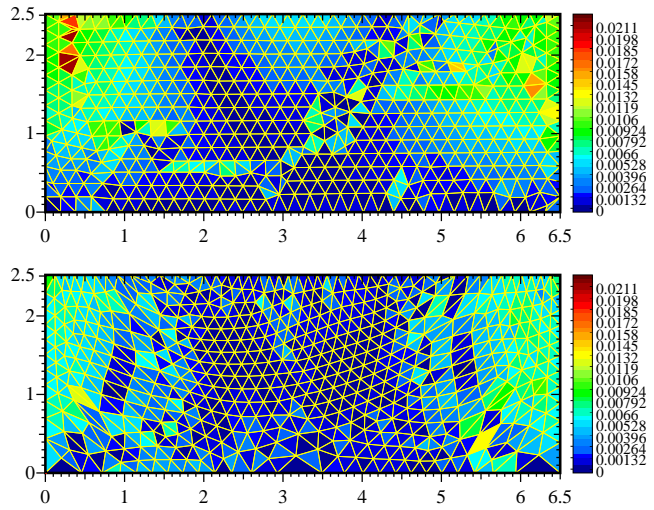

Figure 2: Approximation error distribution on uniform and optimal grids (1000 elements). Darker colors indicate small error.

where

$\hat{A_{i}}=\left[\begin{array}{ccc}\frac{1}{2}\left(\Delta_{1} x_{i}\right)^{2} & \Delta_{1} x_{i} \Delta_{1} y_{i} & \frac{1}{2}\left(\Delta_{1} y_{i}\right)^{2} \\ \vdots & \\ \frac{1}{2}\left(\Delta_{n} x_{i}\right)^{2} & \Delta_{n} x_{i} \Delta_{n} y_{i} & \frac{1}{2}\left(\Delta_{n} y_{i}\right)^{2}\end{array}\right]$ and $\hat{b}_{i}=\left[\begin{array}{c}\Delta_{1} f_{i}-(\nabla \tilde{f})_{1} \cdot \Delta_{1} \mathbf{x}_{i} \\ \vdots \\ \Delta_{n} f_{i}-(\nabla \tilde{f})_{n} \cdot \Delta_{n} \mathbf{x}_{i}\end{array}\right]$,

and

$$
W_{i}=\operatorname{diag}\left(\omega_{i 1}, \cdots, \omega_{i n}\right), \quad \hat{W}_{i}=\operatorname{diag}\left(\hat{\omega_{i 1}}, \cdots, \hat{\omega_{i n}}\right) .
$$

One may obtain, by solving local (usually under-determined) systems for the weights, formulae which would be exact for a conic or a second order polynomial. However in this work, we have used, for simplicity, the weights that would guarantee that property in $1 \mathrm{D}$. The value of such weights is $\omega_{i k}=\left\|\mathbf{x}_{k}-\mathbf{x}_{i}\right\|^{-\frac{3}{2}}$ and $\hat{\omega_{i k}}=1$.

The value of the metric is extended to the whole domain using a $C^{0}$ interpolation. The optimal mesh should satisfy the requirement $\|\mathbf{v}\|_{\mathbf{M}}=1$, with $\mathbf{M}=k|\mathbf{H}|$, for every mesh 
edge v. Clearly, the stated condition may never be satisfied exactly, apart from simple distribution of $\mathbf{M}$ on simple geometries. Therefore, a more appropriate target would be to minimize the function $I=\sum_{\mathbf{v}}\left(\|\mathbf{v}\|_{\mathbf{M}}-1\right)^{2}$.

To this purpose, the approach followed employs the techniques illustrated in [8] which are based on local mesh modification/reconstruction strategies.

In fact, to build an adapted mesh with approximately all edges having the same length in the metric, we have used the following techniques [8] :

- mesh refinement and mesh coarsening (i.e the $h$-method);

- change of node connectivity (diagonal swapping);

- relocalization of the nodes at the "center" of their neighbors (i.e. the r-method).

In particular the mesh is refined by sweeping over all the edges and by doing some local modifications where needed. If the lenght of an edge in larger than a certain fixed lenght, then we cut this edge by introducing a new node and two new edges.

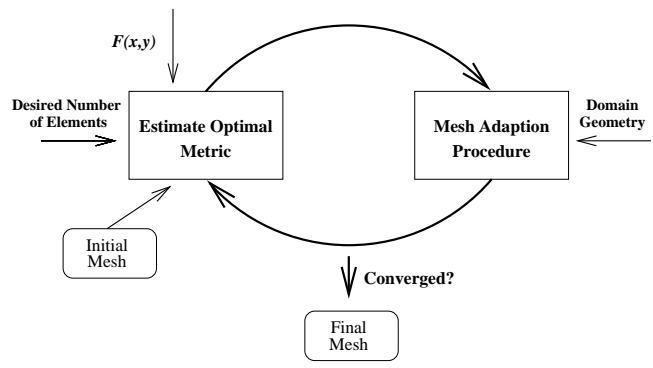

Figure 3: The mesh adaption procedure.

If the lenght of an edge is small respect to the metric, then one of the two nodes of the edge is removed creating a hole in the mesh. This hole is then remeshed. As an edge between two triangles can be seen as a diagonal of a quadrilateral, one can choose between its two diagonals to get the best triangles. An edge-swapping procedure is used as well chosing the configuration which minimizes $\max \left(\mathcal{E}_{K_{1}}, \mathcal{E}_{K_{2}}\right)$, between the two possible configurations.

Also messh movement is implemented. A classical way of moving mesh nodes is to think the mesh as a network of springs. If we want to move a node $x$, all the other nodes are supposed to be fixed. It is easy to determine the new position of the node $x$ minimizing the energy function of the springs problem. Here the spring stiffness has been related to the metric.

All these techniques have been implemented in the code LIBOM (kindly furnished by Prof. Fortin) which has been used as mesh modification procedure in this work.

A preliminary experiment has been carried out by employing an analytic function computing the metric from the exact second derivatives. A rectangular domain $\Omega=[0,6.5] \times[0,2.5]$ has been chosen and the function adopted is $f(x, y)=y \cos (x)+$ $\sin (y)+1$.

The $L_{\infty}$ error norm for an uniform mesh and a mesh computed with the optimal metric has been compared, for different number of elements. Table in figure 1 illustrates the result, while in figure 2 we show the two grids for the 1000 elements case, with the 
$L_{\infty}(K)$ error norm on each element $K$.

It can be noted how the optimal grid displays a more uniform error distribution, some error variation remains due to the approximations made by the mesh generator procedure, which is described in [11].

Then we have experimented the adaptive technique graphically depicted in figure 3. Starting with an initial uniform mesh we have estimated the optimal metric and used the mesh adaption procedure to produce a mesh with the same number of elements but "optimal" with respect to the computed metric. The new grid is then used as new starting mesh for a new adaption iteration. This procedure is strictly analogous to the one often employed in mesh adaption techniques [9].

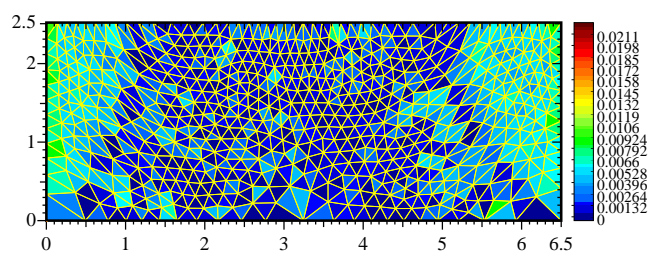

Figure 4: Final grid and error distribution for $f(x, y)=y \cos (x)+\sin (y)+1$ and estimated metric.

The only difference is that here the function is given analytically and it is not the approximated solution of a PDE. The mesh adaption procedure tries to equi-distribute the error by using mesh reconstruction algorithms. Figure 4 illustrates the final grid obtained after 15 adaption iterations for the function $f(x, y)=y \cos (x)+\sin (y)+1$.

The similarity with the analogous mesh of figure 2, obtained using the exact metric, can be noted. The final error distribution shows improvements with respect to the one obtained on the uniform mesh, but it is not so impressive. This is due to two factors. First, the function has moderate second derivatives, therefore the error in the uniform grid was already low. Second, the adaption technique seems to have more difficulties in optimizing the grid near the boundary, where we have an high value for the derivatives and thus of the error.

Therefore, we decided to make a second experiment with a different function, on the same domain,

$$
f\left(x^{*}, y^{*}\right)=\left(x^{* 2}+100 y^{* 2}\right) e^{-5\left(x^{* 2}+y^{* 2}\right)},
$$

where $\left(x^{*}, y^{*}\right)$ have been obtained by shifting the coordinates to have $\left(x^{*}, y^{*}\right)=(0.0)$ at the center of the domain. The uniform grid with 1000 elements and the grid obtained after the adaption procedure (with again approximatively 1000 elements) are shown in figure 5 .

The $L_{\infty}$ norm of the error has dropped from about 1.292 to 0.048 . Figure 6 shows an enlargement of the optimal mesh, where strongly elongated triangles may be found. Finally, figure 7 shows the convergence history iterative procedure for two different functions. In both cases, after just a few iterations, the number of elements and the corresponding error stabilize to their final value. 

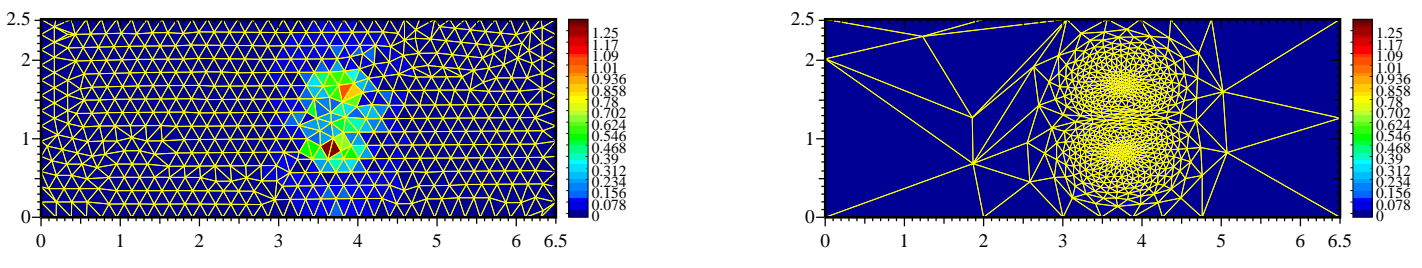

Figure 5: Error distribution on uniform and optimal grids (1000 elements).

\section{Conclusions}

Numerical experiments have shown that adaption techniques based on approximation error estimate and mesh reconstruction procedures may be successfully implemented for creating an optimal mesh for piece-wise linear interpolation. For the cases here examined, the iteration procedure appears to converge to a mesh that nearly equidistributes the approximation error. Some work is still necessary to improve the mesh optimization technique near domain boundaries.

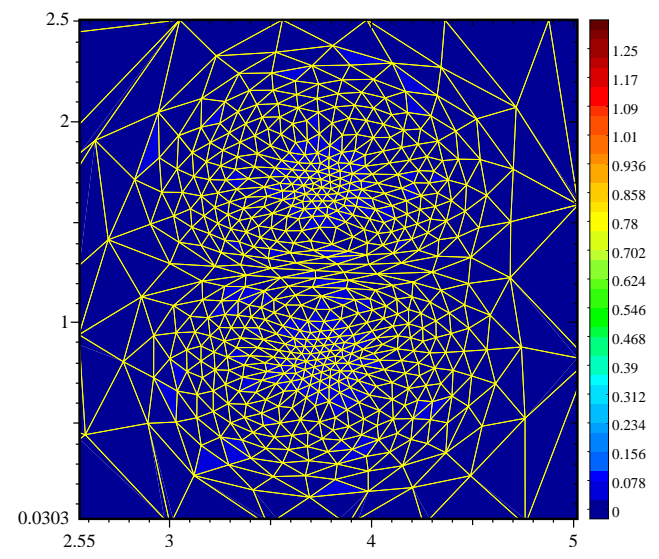

Beside the use in the field of numerical solution of PDE's, the technique can be extended to the problem of finding the best triangulation for a parametric surface. The mesh optimization could take place on the parametric plane and the metric tensor should be modified to account for surface curvature. Moreover, the scheme can be readily expanded to functions in $\mathbf{R}^{3}$ piece-wise linearly approximated on tetrahedral grids.

Figure 6: Enlargement of the optimal mesh

\section{Acknowledgments}

The authors wish to thanks Prof. Fortin from Laval University, Quebec, for the useful discussions we had during his stay at CRS4, and for the availability of the software LIBOM for 2D mesh adaption. This work was supported by the Autonomous Region of Sardinia. 

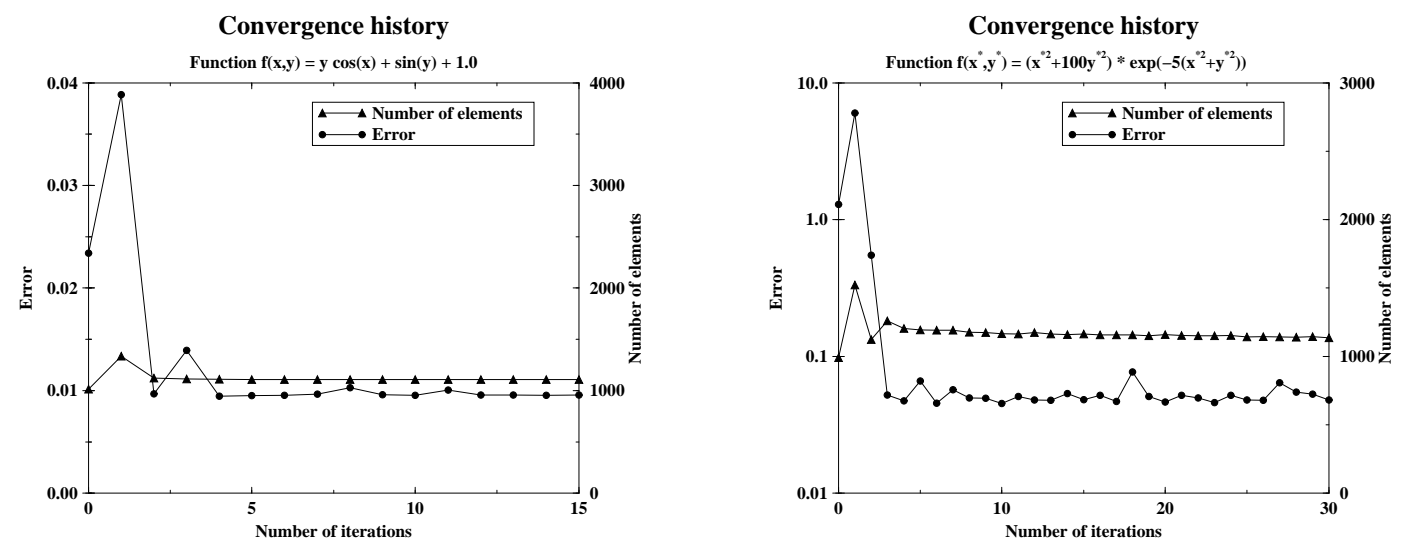

Figure 7: Convergence history of the adaption procedure for 2 different functions. After few iterations of mesh reconstruction, the $L_{\infty}$ norm of the error stabilizes.

\section{REFERENCES}

[1] M. Berzins. Solution-based mesh quality for triangular and tetrahedral meshes. $6^{\text {th }}$ annual international meshing roundtable, 1997, SANDIA Laboratories, Brigham Young University.

[2] H. Borouchaki and P. George and B. Mohammadi. Delaunay mesh generation governed by metric specifications. Part II: Application Examples. Finite Elements in Analysis and Design, 1996, 25, 85-109.

[3] H. Borouchaki and P. L. George and F. Hecht and P. Laug and E. Saltel. Delaunay mesh generation governed by metric specifications. Part I: Algorithms. Finite Elements in Analysis and Design, 1997, 25, 61-83.

[4] E. F. D’ Azevedo. Optimal Triangular Mesh Generation by Coordinate Transformation. SIAM Journal on scientific and statistical computing, 1991.

[5] E. F. D’Azevedo, R. B. Simpson. On Optimal Interpolation Triangle Incidences. SIAM Journal on scientific and statistical computing, 1989.

[6] E. F. D’Azevedo, R. B. Simpson. On Optimal Triangular Meshes for Minimizing the Gradient error. Numerische Mathematik, 1991.

[7] N. Dyn, D. Levin, S. Rippa. Data Dependent Triangulations for Piecewise Linear Interpolation. IMA Journal of Numerical Analysis, 1990.

[8] J. Dompierre, M.-G. Vallet, M. Fortin, W.G. Habashi, D. Aït-Ali-Yahia, S. Boivin, Y. Bourgault, A. Tam. Edge-Based Mesh Adaptation for CFD. CERCA Report R95-73, CERCA, 1995. 
[9] W.G. Habashi, M. Fortin, J. Dompierre, M.-G. Vallet, D. Aït-Ali-Yahia, Y. Bourgault, M.P. Robichaud, A. Tam and S. Boivin. Anisotropic Mesh Optimization for Structured and Unstructured Meshes, 28th VKI CFD Lecture Series, Von Karman Institute for Fluid Dynamics, March 3-7, 1997.

[10] F. Hecht and B. Mohammadi. Mesh Adaption by Metric Control for Multi-scale Phenomena and Turbulence. In AIAA 35th Aerospace Sciences Meeting \& Exhibit, Reno, NV, January 1997. AIAA Paper 97-0859.

[11] L. Formaggia, F. Rapetti. MeSh2d (Unstructured Mesh Generator in 2D), Version 1.0. Algorithm Overview and Description. CRS4 Technical report CRS4-TECHREP-96/4. February 1996.

[12] L. Formaggia, F. Rapetti. MeSh2D (Unstructured Mesh Generator in 2D), Version 1.0. A user's Guide. CRS4 Technical report CRS4-TECH-REP-96-6. February 1996.

[13] L. Formaggia, "Unstructured Mesh Generation and Adaption", in Numerical Analysis, Scientific Computing, Computer Science, Vol. 16, G. Alefeld, O. Mahrenholtz, and R. Mennicken Eds., ZAMM, 1996.

[14] J. A. Gregory. Error Bounds for Linear Interpolation in Triangles. The mathematics of finite elements and application II, Academic Press, 1975.

[15] P. Johnson. Numerical Solution of Partial Differential Equations by the Finite Element Method. Cambridge University Press, 1987.

[16] C. L. Lawson. Software for $C^{1}$ Interpolation. Mathematical Software III. Academic Press, 1977.

[17] E. J. Nadler. Piecewise Linear Approximation on Triangulations of a Planar Region. PhD. Thesis, Div. Applied Math. Brown University. 1985.

[18] S. Rippa. Long and Thin Triangle Can Be Good for Linear Interpolation., SIAM Journal on scientific and statistical computing, 1992.

[19] R. B. Simpson. Anisotropic mesh transformations and optimal error control. Applied Numerical mathematics 14 (1994) 183-198.

[20] M.-G. Vallet. Generation de Maillages Elements Finis Anisotropes et Adaptatifs. $\mathrm{PhD}$ thesis, Universite de Paris VI, 1992. 